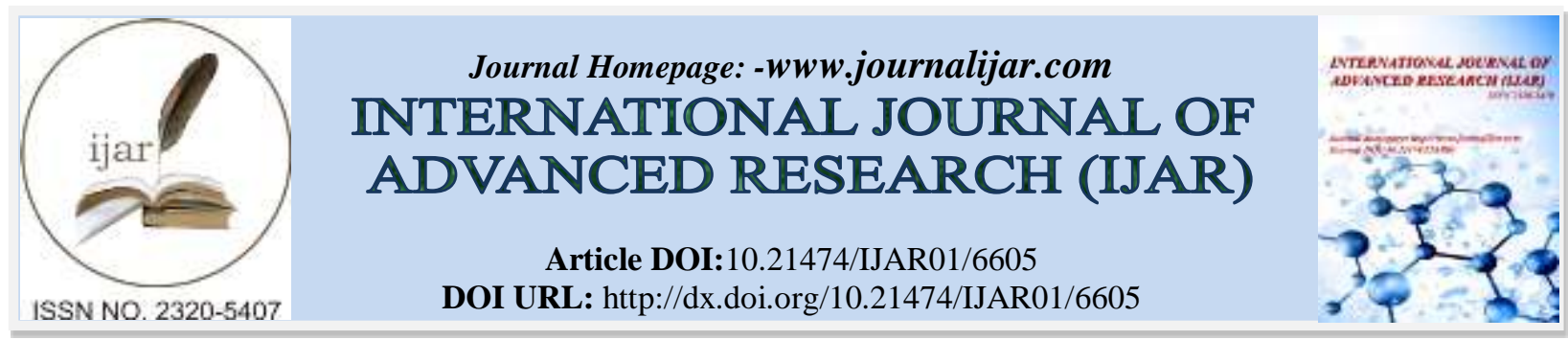

RESEARCH ARTICLE

\title{
PROSPECTIVE STUDY OF ROLE OF CONTRAST SENSITIVITY AND COLOUR VISION IN CENTRAL SEROUS CHORIORETINOPATHY.
}

Dr. G. V. Prasad and Dr. P. Divya.

1. M.D. Ophthalmology, Civil Surgeon, Andhra Medical college, Visakhapatnam.

2. MBBS, Junior Resident, Ophthalmology, Andhra Medical college, Visakhapatnam.

\section{Manuscript Info}

Manuscript History

Received: 21 December 2017

Final Accepted: 23 January 2018

Published: February 2018

\section{Abstract}

Aim: To evaluate Contrast sensitivity and Colour vision during and after resolution of sub retinal fluid in Acute central serous retinopathy and their relation with the Visual acuity(VA) and Central macular thickness(CMT).

Methodology: In this prospective observational study from Jan 2017 sep 2017, 35 patients within the age group of 20-50 years were included. Patients are then divided into 2 groups depending on time taken for absorption of Sub retinal fluid (SRF) .Early resolution group(within 3 months)and delayed resolution group(>3 months), and all the patients included in the study were followed for 6 months.

Results: In the early resolution group $(68.6 \%)$ Best corrected visual acuity( BCVA) 6/6 attained by 2 months, Contrast sensitivity becomes normal by 4.5 months, Colour vision attained normal range by 4 months .

In the Delayed resolution group (31.43\%), BCVA attained by 6months, even in cases with residual SRF. $63.6 \%$ cases showed moderate loss of contrast sensitivity(CS) and tritanopia even after complete resolution of SRF and with normal visual acuity. Persistent SRF cases (3 cases) showed severe colour vision loss and moderate CS loss.

Conclusion: In the early resolution group the recovery of fine vision took about 1 month after resolution of SRF. In the delayed resolution group, the visual acuity showed low rate of improvement, attaining normal range by the end of $4^{\text {th }}$ month and $63.6 \%$ patients showed moderate contrast sensitivity loss even after resolution of SRF and with normal visual acuity. $63.63 \%$ of cases showed colour vision defect by the end of 6th month even after resolution of SRF, and had disturbed vision due to loss of colour and contrast sensitivity, necessitating the need for measures of early intervention like Laser or medical therapy to hasten early resolution of CSC, so that fine vision can be preserved in young working age group especially certain professions like drivers and pilots.

Copy Right, IJAR, 2018,. All rights reserved. 


\section{Introduction:-}

Central serous chorioretinopathy(CSC) is a disorder characterised by detachment of the neurosensory retina from the underlying retinal pigment epithelium at the posterior pole ${ }^{(1)}$. It is generally seen in $2^{\text {nd }}-3^{\text {rd }}$ decade males unilaterally. Rarely bilateral cases are also seen ${ }^{(2)}$.

Patients have varied symptomatic profile, starting from asymptomatic to sudden onset of blurring of vision, metamorphopsia, central or paracentral scotoma ${ }^{(1,2)}$ The visual acuity differs among the patients but usually presents with hypermetropic refractive error ${ }^{(3)}$. Visual disturbance still persists in patients even after giving the best correction, which highlights the need for the evaluation of the fine vision i.e., contrast sensitivity and colour vision in the patients of CSC.

CSC is a self limiting disorder in most of the cases with spontaneous resolution of subretinal fluid within 3-4 months ${ }^{(1-4)}$ Even though the initial visual acuity has been attained by most of these cases, there are still patients with disturbance in vision. Many studies evaluated the visual acuity and central macular thickness (CMT) after the resolution of subretinal fluid ${ }^{.(5)}$ but there are limited studies on contrast sensitivity and colour vision during and after resolution of SRF in Acute $\mathrm{CSC}^{\cdot(6)}$

\section{Methodology:-}

In this prospective observational study conducted at tertiary care hospital, visakhapatnam, from Jan 2017 - sep 2017, 35 patients presented between jan 2017 - April 2017, within the age group of 20-50 years were included. The patients presented to the OPD with visual complaints $<3$ months and fundus features suggestive of CSC, which was confirmed by Fundus fluorescein angiography (FFA).

The patients with other retinal diseases, previous ocular surgery within 6 months, media opacity, fellow eye with poor visual acuity, patients who had macular laser treatment were excluded from the study. Informed consent was taken.

The Patients were evaluated at baseline and followed up each month. At each visit, the examination included: (1)Best corrected visual acuity (BCVA) by Snellens chart and converted to decimal equivalents, contrast sensitivity measured by Pelli Robson chart and colour vision by Ishihara plates (17/17) and Farnsworth D -15 ; (2) Measurement of central macular thickness (CMT) by HD- OCT (3) Fluorescein angiography (FA) was performed in all patients at baseline and after 3 months in eyes with persistence or recurrence of SRF.

The pattern of the contrast sensitivity, colour vision and BCVA with respect to the central macular thickness(SRF) were assessed and data were analysed using statistical tests.All the patients were followed for 6 months.

Results were calculated as mean \pm Standard deviation (SD)/median for age, duration of symptoms, OCT values and percentages for gender, laterality, angiographic features and visual outcome. Association of various variables with final visual outcome was analysed using Chi square test/Fischer exact test. Paired ' $t$ ' test was used to compare initial and final BCVA, initial and final contrast sensitivity values. A p $<0.05$ was considered significant.

\section{Results:-}

In this study, the mean age of the patients is 33.4 years. All the recorded cases are of male gender (100\%), with 28 patients $(80 \%)$ unilateral presentation and 7 patients $(20 \%)$ are bilateral.

The fundus fluorescence angiography at the baseline showed single leaks in 30 cases, two leaks in 4 patients and multiple leaks in 1 patient. Ink blot pattern was observed in 25(62.5\%) and smoke stack pattern in 15(37.5\%).

The patients are grouped into 2 groups based on their time taken for resolution of sub retinal fluid (SRF).

Early resolution group(within 3 months) -24 patients.

Delayed resolution group (> 3months) -11 patients. 


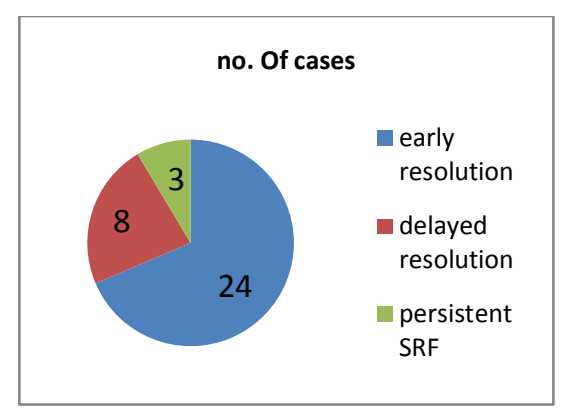

Chart 1:- shows no. Of cases in each group

Early resolution group: 24 patients:-

The patients in the early resolution group showed statistically significant improvement of visual acuity in relation with the central macular thickness. The visual acuity returned to the 6/6 on Snellens chart. 7 cases showed Pigment epithelial detachment within SRF, did not effect the visual acuity after resolution.

All the patients in this group showed gross defect in colour vision at the baseline. At 2 months, 21 out of 24 cases remained defective, majority of them showed tritanopia, remaining 3 cases had normal colour vision. at the end of 3 months, all the 19 cases showed blue colour deficiency, 5 cases showed normal colour vision. Even after complete resorption of SRF, 19 out of 24 cases showed colour vision defect. it took about mean of 1.5 months after the resorption of SRF for the colour vision to become normal.

The patients had showed significant improvement of contrast sensitivity with the decrease of the Central macular thickness(along with SRF). The patients showed severe contrast sensitivity loss at the baseline. The mean CS at the base line was $0.35 \log$ units. The CS at the 2 months was 0.90 and at the end of 5 months, the CS returned to normal.Even though these patients had good visual acuity at the end of 2 months, these patients achieved normal contrast sensitivity at 4 months. The rate of gaining the contrast sensitivity is more in the first 2 months than in the next 3 months.

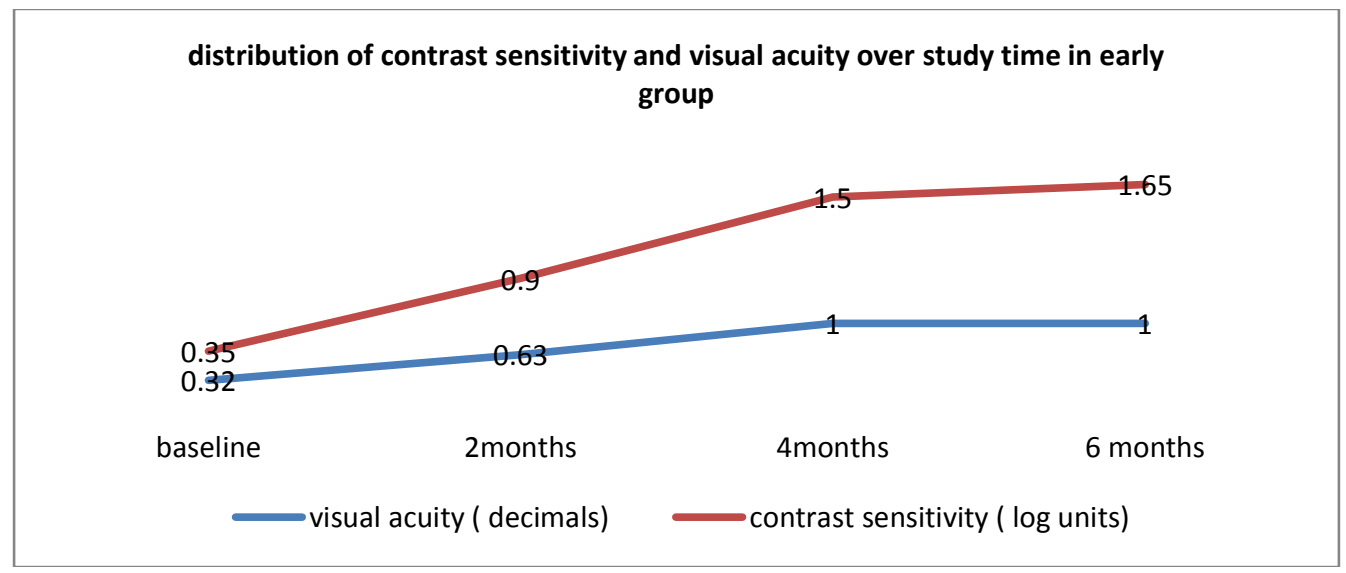

Chart 2:- distribution of CS and VA over 6 months in early resolution group

\section{Delayed resolution group : 11 patients:-}

The patients in the delayed resolution group showed statistically significant improvement of visual acuity in relation with the central macular thickness. The visual acuity returned to the 6/6 on Snellens chart at the end of 6 months in all the cases, even when there is residual SRF thickness in 3 cases.

All the patients in this group showed defective colour vision at the baseline. At 2 months. patients colour vision remained grossly defective. At the end of 4 months, 8 cases showed tritanopia, 3 showed gross defect in colour vision. At 6 months, 7 cases showed tritanopia , 3 cases showed gross defective color vision and 1 case showed normal vision 
The patients had showed significant improvement of contrast sensitivity with the decrease of the Central macular thickness(along with SRF). The patients showed severe contrast sensitivity loss at the baseline and at 2 months. The mean CS at the base line was $0.15 \log$ units. The CS at the 2 months was $0.23 \log$ units. The CS at 4 months is 0.88 $\log$ units. The CS at 5 months is $1.27 \log$ units. At the end of 6 months, the mean CS is at $1.55 \log$ units for 7 cases, even with complete resorption of SRF and the remaining 4 cases showed normal CS by 6 months. The contrast sensitivity improvement is slow than the visual acuity and colour vision gain in this group.

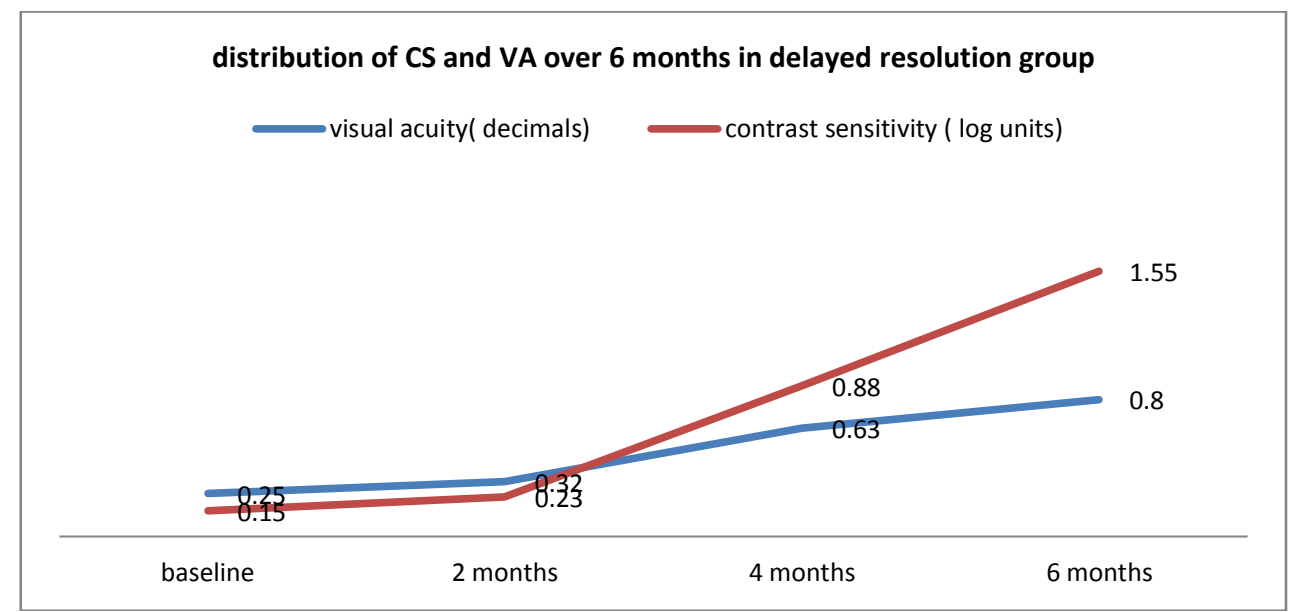

Chart 3:- distribution of CS and VA over 6 months in delayed resolution group

Comparison of contrast sensitivity between the two groups showed the difference in the gain in contrast sensitivity and also the rate of gain., which is less in delayed resolution group compared to the early group. The visual acuity gain also showed significant difference between the two groups.

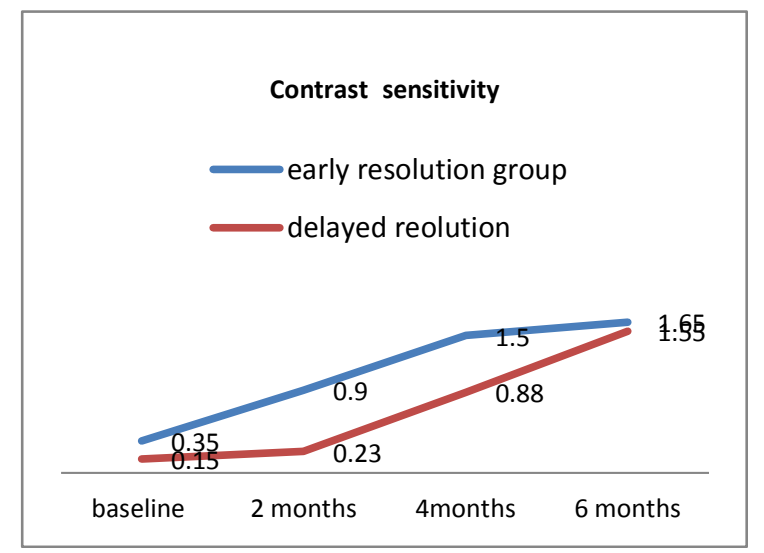

Chart 4:- comparison of contrast sensitivity gain between the early resolution group and delayed resolution group

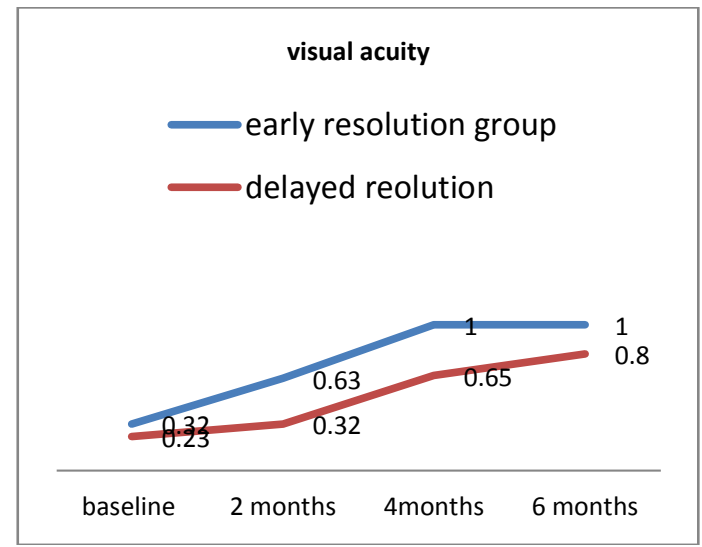

Chart 5:- comparison of visual acuity gain between the early resolution group and delayed resolution group

\section{Discussion:-}

This study examined the course of contrast sensitivity, colour vision and visual acuity in patients with acute central serous chorioretinopathy (onset of symptoms within 10 days $-<1$ month). The majority of the cases included in the study were presented within 10-15 days of onset of the symptoms. These cases were studied up to 6 months in order to note the patterns of visual parameters and also to note the recurrences if any.

Majority of the cases (19 of 35) had complete resolution of sub retinal fluid (SRF) within 2 months which is in comparison with the previous literature. 11 cases showed persistent SRF at the end of 3 months, these were grouped under delayed resolution. The resolution rate is low in delayed group which is spanned between 3-6months. 3 of 35 
cases showed residual SRF(minimal) at the end of 6 months for which they were subjected to the treatment according to the hospital protocol.

\section{Visual acuity:-}

$61.3 \%$ of patients in the study presented with the central scotoma, with gross central blurring of vision at the baseline, which has improved significantly in the first 2 months in $60 \%$. This has improved to a $80 \%$ by the end of $4^{\text {th }}$ month. By 6 th month there is $97 \%$ of visual acuity gain. The visual acuity gain is statistically significant with the resolution of the SRF.

In early resolution group, all patients showed total visual acuity gain by the end of 3 months, with $79 \%$ showing $6 / 6$ vision by the end of $2^{\text {nd }}$ month. When compared to this, the delayed resolution group showed a low rate of visual acuity gain. 4 out of 11 patients in delayed group showed $6 / 6$ vision at the end of $4^{\text {th }}$ month. At 6 months, all patients of the group had 6/6 vision. 3 patients with persistent SRF had near normal vision.

\section{Contrast sensitivity:-}

In the early group, the patients had severe contrast sensitivity loss at the baseline with the mean of $0.35 \log$ units. We observed a rapid gain of CS in the first 2 months along with visual acuity. The CS at 2 months is $0.90 \log$ units which showed moderate loss of CS. Even though majority of patients had normal visual acuity at this time they still had loss of contrast sensitivity. By the end of 3 months all the patients had complete resorption of the SRF, with 6/6 visual acuity and mean contrast sensitivity of $1.12 \mathrm{log}$ units, showing a slight reduction in CS when compare with visual acuity in this group. All the patients in this group showed normal CS by $4{ }^{\text {th }}$ month.

In the delayed group, all the patients had severe loss of CS at the baseline, which had improved with the visual acuity upto 4 months. The mean CS at the end of 2 months still showed severe CS loss when compared to the early group. The CS started to improve at 4months and gradual gain has noted till 6 months. The gain is slow when compared with the visual acuity gain in the same group and with CS gain in the early resolution group. There is a moderate CS loss in cases with complete resolution of SRF which is high when compared to early resolution group. At the end of 6 months, 50\% cases showed moderate loss of CS even with no residual SRF. All the 3 cases with persistent SRF, near normal visual acuity by 6 months showed moderate loss of contrast sensitivity . these $63.6 \%$ patients in the delayed group were not satisfied with their visual acuity even if $6 / 6$ on Snellens chart. They still complained about the visual disturbance., this study showed similar results of contrast sensitivity loss with other studies in literature. ${ }^{(6,7,8)}$

This shows the importance of measuring the contrast sensitivity along with visual acuity at each visit in the patients in acute onset of central serous chorioretinopathy.

\section{Color vision:-}

In the present study, all the 35 patients showed gross defective vision at the baseline.

In the early resolution group, at the end of 2 months $87.5 \%$ patients had tritanopia, remaining $12.5 \%$ cases had normal colour vision. At the end of 3 months, $79.16 \%$ showed blue colour deficiency, $28.8 \%$ cases showed normal colour vision. Even after complete resorption of SRF, 79\% cases showed colour vision defect. it took about mean of 1.5 months after the resorption of SRF for the colour vision to become normal.

In delayed resolution group, all the patients in this group showed defective colour vision at the baseline and at 2 months. At the end of 4 months, $72.7 \%$ cases had improvement in colour vision but still showed tritanopia while $27.27 \%$ showed gross defect in colour vision. At the end of 6 months, $63.63 \%$ cases with complete resolution of SRF showed tritanopia, remaining cases gained near normal colour vision. The cases with persistent SRF showed severe colour vision loss by 6 months with little improvement.

The results from this study are similar to the studies conducted by Spitznas M et al. ${ }^{(9)}$ In a study by Maaranen TH et al., ${ }^{(10)}$ it showed that of the CSR eyes $67 \%$ had a colour vision defect, with majority of them having tritanopia.

Limitations of the study: Small sample size of the delayed resolution group, in order to get a more statistically significant data we need a larger sample size. This study showed that much longer follow up period is needed to get more information about the contrast sensitivity and colour vision course in the cases with CSR. 


\section{Conclusion:-}

In the Early resolution group, even after visual acuity is normal, the fine vision parameters like contrast sensitivity and colour vision are still reduced and it takes about 1 month after resolution of SRF for recovery.

In the Delayed resolution group, the visual acuity showed low rate of improvement attaining normal range by the end of $4^{\text {th }}$ month. 50\% patients showed moderate contrast sensitivity loss even after resolution of SRF and with normal visual acuity. $63.63 \%$ of cases showed colour vision defects by the end of 6 month even after resolution of SRF.

With the normal visual acuity, $63.6 \%$ of the patients had disturbed vision due to loss of colour and contrast sensitivity and are not happy with the vision.

This study highlights the need for measurement of colour vision and contrast sensitivity and aim for early intervention for the resolution of SRF in CSR cases, as the patients affected by CSR are in productive age group i.e.., 25-35 years, and the need for fine vision for their working lifestyle.

\section{References:-}

1. Nicholson B, Noble J, Forooghian F, Meyerle C. Central serous chorioretinopathy: update on pathophysiology and treatment. Survey of Ophthalmology. 2013, 58(2): 103- 126

2. Liegl R, Ulbig M W. Central serous chorioretinopathy. Ophthalmologica. Journal International d'ophtalmologie. International journal of ophthalmology. Zeitschrift fur Augenheilkunde. 2014, 232: 65-76.

3. Structural and Functional Outcomes in Chronic Central Serous Chorioretinopathy Treated with Photodynamic Therapy Pino Cidad, Eugenia González, Mónica Asencio, Jesús García Korean J Ophthalmol 2015;29(5):331335 http://dx.doi.org/10.3341/kjo.2015.29.5.331

4. OCT Angiography Compared to Fluorescein and Indocyanine Green Angiography in Chronic Central Serous Chorioretinopathy.

5. Teussink MM, Breukink MB, van Grinsven MJ, Hoyng CB, Klevering BJ, Boon CJ, de Jong EK, Theelen TInvest Ophthalmol Vis Sci. 2015 Aug; 56(9):5229-37.

6. Clin Ophthalmol. 2012;6:1949-54. doi: 10.2147/OPTH.S38363. Epub 2012 Nov 26.Correlation of spectral domain optical coherence tomography findings in acute central serous chorioretinopathy with visual acuity. Nair U1, Ganekal S, Soman M, Nair K.

7. Pattern of Contrast Sensitivity Changes in Acute Central Serous Chorioretinopathy Hindawi Journal of Ophthalmology Volume 2017, Article ID 9053932, 8 pages https://doi.org/10.1155/2017/9053932.

8. Graefes Arch Clin Exp Ophthalmol. 1994 Aug;232(8):473-6. Contrast sensitivity after resolution of central serous retinopathy. Koskela $\mathrm{P}^{1}$, Laatikainen L, von Dickhoff K.

9. Maaranen T, Mantyjarvi M. Contrast sensitivity in patients recovered from central serous chorioretinopathy. Int Ophthalmo 1999; 23: 31-35

10. Spitznas M. Pathogenesis of central serous retinopathy: a new working hypothesis. Graefe's Arch Clin Exp Ophthalmol 1986;224(4):321-324.

11. Maaranen TH, Tuppurainen KT, Mantyjarvi MI. Color vision defects after central serous chorioretinopathy. Retina 2000;20(6):633-637. 